\title{
Liver injury in critically ill patients with COVID-19: a case series
}

\author{
Filipe S. Cardoso ${ }^{*}$ (D), Rui Pereira and Nuno Germano
}

Dear Editor,

Almost all reports on liver injury in patients with 2019 coronavirus disease (COVID-19) found blood liver tests to be frequently abnormal, especially in patients with more severe disease, but with substantial heterogeneity [1]. Moreover, blood liver tests' abnormalities were frequently thought to be of doubtful clinical value.

Most studies have described blood liver tests in a single time point, usually at inclusion $[2,3]$. Therefore, we used our case series of the first 20 consecutive patients with COVID-19 admitted to the intensive care unit (ICU) at Curry Cabral Hospital in Lisbon, Portugal, from March 10, 2020, onwards, to describe the temporal evolution of blood liver tests.

Median (interquartile range (IQR)) age was 67 (5274) years with 18 (90\%) males (Table 1). Median (IQR) time from symptom onset to hospital admission was 7.5 (5.5-8.5) days, and median time from hospital admission to ICU admission was $1.1(0.7-2.1)$ days. All patients required invasive mechanical ventilation on ICU admission. Median (IQR) sequential organ failure assessment (SOFA) score on ICU admission and peak was 8 (7-9) and $9(8-11)$, respectively. As of April 10, following a median (IQR) of 21.5 (11.225.4) days post ICU admission, 3 (15\%) patients died of multi-organ failure, $14(70 \%)$ were discharged to the ward, and $3(15 \%)$ remained in the ICU.

No patient had documented liver disease prior to hospitalization. During the first 10 days post ICU admis- sion, all patients had at least one abnormal blood liver test. Overtime, only median gamma-glutamiltranspeptidase (GGT), alanine transferase (ALT), and aspartate transferase (AST) showed any increase from upper limit of normal (ULN) and only median GGT had a $\geq$ 3 fold increase from ULN (Table 2). Median peak GGT was on day 8 post ICU admission. Patients with peak C-reactive protein $\geq 250 \mathrm{mg} / \mathrm{L}$ (day 4 post ICU admission) had higher but non-significant median peak GGT ( 298 vs. $125 \mathrm{IU} / \mathrm{L})$, ALT (101 vs. $42 \mathrm{IU} / \mathrm{L}$ ), or AST (72 vs. $57 \mathrm{IU} / \mathrm{L})$ than others $(P>0.50$ for all comparisons).

In our case series, liver injury was frequent but generally transient and non-severe. While synthetic function was largely preserved, late cholestasis was frequently observed. Cholestasis may have been associated to the critical illness itself (inflammation), parenteral nutrition (only 2 patients required parenteral nutrition), or drug toxicity (all patients were on antibiotics, for example ceftriaxone, amoxicillinclavulanate, or azithromycin) [4,5]. While cholestasis could have been multifactorial, the differential diagnosis was not easy to perform with precision. Overall, attention to modifiable factors, such as control of inflammation, timing of parenteral nutrition, and avoiding drugs with worse liver toxicity profile, may be important to prevent cholestasis progression in these patients. Further studies are needed to understand liver injury in critically ill patients with COVID-19, especially if there is any direct viral effect on the liver cells.

\footnotetext{
* Correspondence: filipe_sousacardoso@hotmail.com Intensive Care Unit, Curry Cabral Hospital, R. Beneficiencia N8, 1050-099 Lisbon, Portugal
} 
Table 1 Baseline characteristics and outcomes of patients

\begin{tabular}{ll}
\hline Characteristic & Median (IQR) or $n(\%)$ \\
\hline Age (years) & $67(52-74)$ \\
Sex (male) & $18(90 \%)$ \\
$\mathrm{BMI}\left(\mathrm{kg} / \mathrm{m}^{2}\right)$ & $29(26-32)$ \\
Parameters on ICU admission & \\
$\mathrm{P}_{\mathrm{a}} \mathrm{O}_{2} / \mathrm{FiO} \mathrm{O}_{2}$ & $138(128-163)$ \\
Lactate (mmol/L) & $1.1(0.8-1.2)$ \\
Creatinine (mg/dL) & $1.11(1.04-1.26)$ \\
Organ support during ICU stay & \\
Invasive mechanical ventilation & $20(100 \%)$ \\
Vasopressors & $19(95 \%)$ \\
Renal replacement therapy & $7(35 \%)$ \\
SOFA score & \\
ICU admission & $8(7-9)$ \\
Peak & $9(8-11)$ \\
ICU discharge & $3(2-6)$ \\
APACHEIl score & $18(14-21)$ \\
ICU mortality & $3(15 \%)$ \\
Hospital mortality & $3(15 \%)$ \\
ICU length of stay (days) & $10.3(8.0-12.3)$ \\
Hospital length of stay (days) & $22.4(14.1-26.7)$ \\
\hline
\end{tabular}

IQR interquartile range, $B M I$ body mass index, $\mathrm{P}_{a} \mathrm{O}_{2} / \mathrm{FiO} 2$ oxygen partial pressure/oxygen inspired fraction, SOFA Sequential Organ Failure Assessment, ICU intensive care unit, APACHEII Acute Physiology and Chronic Health Evaluation II

Table 2 Temporal evolution of median levels of blood tests

\begin{tabular}{|c|c|c|c|c|c|c|c|c|c|c|c|}
\hline $\begin{array}{l}\text { Test (upper } \\
\text { limit of } \\
\text { normal) }\end{array}$ & $\begin{array}{l}\mathrm{H} \\
\mathrm{adm}\end{array}$ & $\begin{array}{l}\mathrm{ICU} \\
\mathrm{adm}\end{array}$ & D2 & D3 & D4 & D5 & D6 & D7 & D8 & D9 & D10 \\
\hline INR $(\leq 1.2)$ & 1.18 & 1.17 & 1.20 & 1.16 & 1.17 & 1.18 & 1.17 & 1.15 & 1.17 & 1.17 & 1.16 \\
\hline $\begin{array}{l}\text { Bilirubin } \\
(\leq 1.2 \mathrm{mg} / \mathrm{dL})\end{array}$ & 0.65 & 0.80 & 1.01 & 1.16 & 0.80 & 0.86 & 1.05 & 0.92 & 1.15 & 0.87 & 0.97 \\
\hline $\begin{array}{l}\text { ALP } \\
(\leq 150 \mid \cup / L)\end{array}$ & 61 & 59 & 61 & 74 & 83 & 89 & 101 & 95 & 116 & 125 & 111 \\
\hline $\begin{array}{l}\text { GGT } \\
(\leq 64 \mathrm{IU} / \mathrm{L})\end{array}$ & 55 & 52 & 53 & 66 & 92 & 73 & 102 & 214 & 237 & 211 & 225 \\
\hline $\begin{array}{l}\mathrm{ALT} \\
(\leq 55 \mathrm{IU} / \mathrm{L})\end{array}$ & 31 & 30 & 33 & 31 & 43 & 48 & 56 & 67 & 82 & 55 & 72 \\
\hline $\begin{array}{l}\text { AST } \\
(\leq 34 \mathrm{IU} / \mathrm{L})\end{array}$ & 51 & 51 & 51 & 44 & 49 & 57 & 69 & 62 & 60 & 53 & 46 \\
\hline $\begin{array}{l}\text { CRP } \\
(\leq 5 \mathrm{mg} / \mathrm{L})\end{array}$ & 176 & 207 & 239 & 257 & 271 & 258 & 198 & 153 & 97 & 76 & 74 \\
\hline
\end{tabular}

$\mathrm{H}$ adm hospital admission, ICU adm intensive care unit admission, INR international normalized ratio, ALP alkaline phosphatase, GGT gammaglutamiltranspeptidase, $A L T$ alanine aminotransferase, AST aspartate aminotransferase, CRP C-reactive protein

\section{Abbreviations}

ALT: Alanine transferase; ALP: Alkaline phosphatase; AST: Aspartate transferase; COVID-19: 2019 coronavirus disease; CRP: C-reactive protein; ICU: Intensive care unit; IMV: Invasive mechanical ventilation; INR: International normalized ratio; IQR: Interquartile range; GGT: Gammaglutamiltranspeptidase

\section{Acknowledgements}

Not applicable.

\section{Authors' contributions}

FSC collected, analyzed, and interpreted the data. FSC drafted the manuscript. The authors read and approved the final manuscript.

\section{Funding}

The authors declare that they have no funding source.

\section{Availability of data and materials}

The datasets generated and/or analyzed during the current study are not publicly available due to confidentiality but are available from the corresponding author on reasonable request.

\section{Ethics approval and consent to participate}

The ethics committee at Curry Cabral Hospital, Lisbon, Portugal, waived the need for consent because the study was observational.

\section{Consent for publication}

Not applicable.

\section{Competing interests}

The authors declare that they have no competing interests.

Received: 17 April 2020 Accepted: 27 April 2020

Published online: 05 May 2020

\section{References}

1. Bangash MN, Patel J, Parekh D. COVID-19 and the liver: little cause for concern. Lancet Gastroenterol Hepatol. 2020. https://doi.org/10.1016/S24681253(20)30084-4.

2. Arentz M, Yim E, Klaff L, Lokhandwala S, Riedo FX, Chong M, et al. Characteristics and outcomes of 21 critically ill patients with COVID-19 in Washington State. JAMA. 2020. https://doi.org/10.1001/jama.2020.4326.

3. Bhatraju PK, Ghassemieh BJ, Nichols M, Kim R, Jerome KR, Nalla AK, et al. Covid-19 in critically ill patients in the Seattle region - case series. N Engl J Med. 2020. https://doi.org/10.1056/NEJMoa2004500.

4. Vanwijngaerden YM, Wauters J, Langouche L, Vander Perre S, Liddle C, Coulter $S$, et al. Critical illness evokes elevated circulating bile acids related to altered hepatic transporter and nuclear receptor expression. Hepatology. 2011;54(5):1741-52.

5. Jenniskens M, Langouche L, Van den Berghe G. Cholestatic alterations in the critically ill: some new light on an old problem. Chest. 2018; 153(3):733-43.

\section{Publisher's Note}

Springer Nature remains neutral with regard to jurisdictional claims in published maps and institutional affiliations.

Ready to submit your research? Choose BMC and benefit from:

- fast, convenient online submission

- thorough peer review by experienced researchers in your field

- rapid publication on acceptance

- support for research data, including large and complex data types

- gold Open Access which fosters wider collaboration and increased citations

- maximum visibility for your research: over $100 \mathrm{M}$ website views per year

At BMC, research is always in progress.

Learn more biomedcentral.com/submissions 